\title{
Proposal of a Preoperative CT-Based Score to Predict the Risk of Clinically Relevant Pancreatic Fistula after Cephalic Pancreatoduodenectomy
}

\author{
Marius Lucian Savin 1,2 ${ }^{1}$, Florin Mihai 1,2, Liliana Gheorghe 1,2, Corina Lupascu Ursulescu 1,2,*, \\ Dragos Negru 1,2,*, Ana Maria Trofin ${ }^{1,3}$, Mihai Zabara ${ }^{1,3}$, Vlad Nutu ${ }^{1,3}$, Ramona Cadar ${ }^{3}$, Mihaela Blaj ${ }^{1,4}$, \\ Oana Lovin ${ }^{4}$, Felicia Crumpei ${ }^{3}$ and Cristian Lupascu ${ }^{1,3}$
}

1 “Gr. T. Popa” University of Medicine and Pharmacy, 700115 Iasi, Romania; marius-lucian.savin@umfiasi.ro (M.L.S.); florin.g.mihai@umfiasi.ro (F.M.); liliana.gheorghe@umfiasi.ro (L.G.); ana-maria.trofin@umfiasi.ro (A.M.T.); mihai-lucian.zabara@umfiasi.ro (M.Z.); nutu.vlad@umfiasi.ro (V.N.); mihaela.blaj@umfiasi.ro (M.B.); cristian.lupascu@umfiasi.ro (C.L.)

2 Department of Radiology, “St. Spiridon” Emergency Hospital, 700111 Iasi, Romania

3 Department of Surgery, "St. Spiridon" Emergency Hospital, 700111 Iasi, Romania; ramona-petronela-cadar@email.umfiasi.ro (R.C.); felicia.crumpei@umfiasi.ro (F.C.)

4 Department of Anesthesiology and Intensive Care, “St. Spiridon” Emergency Hospital, 700111 Iasi, Romania; oana.apopei@umfiasi.ro

* Correspondence: corina.ursulescu@umfiasi.ro (C.L.U.); dragos.negru@umfiasi.ro (D.N.)

Citation: Savin, M.L.; Mihai, F.; Gheorghe, L.; Lupascu Ursulescu, C.; Negru, D.; Trofin, A.M.; Zabara, M.; Nutu, V.; Cadar, R.; Blaj, M.; et al. Proposal of a Preoperative CT-Based Score to Predict the Risk of Clinically Relevant Pancreatic Fistula after Cephalic Pancreatoduodenectomy. Medicina 2021, 57, 650. https:// doi.org/10.3390/medicina57070650

Received: 17 May 2021

Accepted: 21 June 2021

Published: 24 June 2021

Publisher's Note: MDPI stays neutral with regard to jurisdictional claims in published maps and institutional affiliations.

Copyright: (c) 2021 by the authors. Licensee MDPI, Basel, Switzerland. This article is an open access article distributed under the terms and conditions of the Creative Commons Attribution (CC BY) license (https:// creativecommons.org/licenses/by/ $4.0 /)$.

\begin{abstract}
Background and Objectives: Postoperative pancreatic fistula after cephalic pancreatoduodenectomy (CPD) is still the leading cause of postoperative morbidity, entailing long hospital stay and costs or even death. The aim of this study was to propose the use of morphologic parameters based on a preoperative multisequence computer tomography (CT) scan in predicting the clinically relevant postoperative pancreatic fistula (CRPF) and a risk score based on a multiple regression analysis. Materials and Methods: For 78 consecutive patients with CPD, we measured the following parameters on the preoperative $\mathrm{CT}$ scans: the density of the pancreas on the unenhanced, arterial, portal and delayed phases; the unenhanced density of the liver; the caliber of the main pancreatic duct (MPD); the preoperatively estimated pancreatic remnant volume (ERPV) and the total pancreatic volume. We assessed the correlation of the parameters with the clinically relevant pancreatic fistula using a univariate analysis and formulated a score using the strongest correlated parameters; the validity of the score was appreciated using logistic regression models and an ROC analysis. Results: When comparing the CRPF group (28.2\%) to the non-CRPF group, we found significant differences of the values of unenhanced pancreatic density (UPD) $(44.09 \pm 6.8 \mathrm{HU}$ vs. $50.4 \pm 6.31 \mathrm{HU}, p=0.008)$, delayed density of the pancreas ( $48.67 \pm 18.05 \mathrm{HU}$ vs. $61.28 \pm 16.55, p=0.045)$, unenhanced density of the liver (UDL) $(44.09 \pm 6.8 \mathrm{HU}$ vs. $50.54 \pm 6.31 \mathrm{HU}, p=0.008), \mathrm{MPD}(0.93 \pm 0.35 \mathrm{~mm}$ vs. $3.14 \pm 2.95 \mathrm{~mm}, p=0.02)$ and ERPV $\left(46.37 \pm 10.39 \mathrm{~cm}^{3}\right.$ vs. $\left.34.87 \pm 12.35 \mathrm{~cm}^{3}, p=0.01\right)$. Based on the odds ratio from the multiple regression analysis and after calculating the optimum cut-off values of the variables, we proposed two scores that both used the MPD and the ERPV and differing in the third variable, either including the UPD or the UDL, producing values for the area under the receiver operating characteristic curve (AUC) of 0.846 (95\% CI $0.694-0.941)$ and $0.774(95 \%$ CI $0.599-0.850)$, respectively. Conclusions: A preoperative CT scan can be a useful tool in predicting the risk of clinically relevant pancreatic fistula.
\end{abstract}

Keywords: clinically relevant pancreatic fistula; pancreatic volumetry; computer tomography; pancreatoduodenectomy

\section{Introduction}

Despite the many advances in preoperative imaging, refinements of the operative technique and postoperative management, pancreatic fistula $(\mathrm{PF})$ remains the main and 
feared complication after cephalic pancreatoduodenectomy (CPD), causing high morbidity (i.e., high risk of acute pancreatitis, hemorrhage and severe peritonitis) and a prolonged hospital stay [1-3].

Previous studies identified several risk factors for PF, including a soft pancreas (lipomatosis), hard pancreas (fibrosis), Wirsung duct caliber and obesity $[4,5]$.

The consistence of the pancreas is a subjectively assessed intraoperative parameter that is determined by the balance between the lipomatosis and fibrosis and affects the quality of the pancreatoenteric suture [6,7]. Although the pathologic assessment is the most accurate in determining the parameters of the texture of the pancreas, a preoperative multisequence computer tomography (CT) can determine the degree of fatty infiltration of the pancreas and the fibrosis, based on the unenhanced density and the pattern of enhancement of the pancreas [8-11].

A high caliber of the main pancreatic duct favors a good pancreaticojejunal anastomosis and reduces the risk of PF, provided there is a duct-to-mucosa anastomosis [12].

The pancreatic remnant volume has been proven to independently predict the risk of clinically relevant pancreatic fistula [13].

The aim of the study was to assess the capability of independent parameters evaluated on a preoperative CT scan to predict the clinically relevant pancreatic fistula and to draw up a score based on a logistic regression analysis to detect high-risk patients.

\section{Materials and Methods}

\subsection{Study Design and Patients}

This retrospective study was approved by the Bioethics Committee of "Gr. T. Popa", University of Medicine Iasi (No.19802, approved date: 14 April 2020). We searched our prospectively maintained database for patients who underwent CPD between 2015 and 2020 that also had an available preoperative multisequence CT scan of the abdomen. The CPD was performed for preoperative high suspicion of pancreatic head cancer or periampullary tumors. No distant metastases or borderline resectable tumors were noted preoperatively. We excluded patients where the marked atrophy of the pancreas did not allow the drawing of a sufficiently large region of interest (ROI) to determine the density of the pancreas and where the pancreatic resection was not performed at the level of the left margin of the superior mesenteric vein (SMV).

All the patients included in the study were represented by randomized numeric codes, and all personal data were hidden.

\subsection{Surgical Procedure for $C P D$}

The surgical procedure consisted of a backwards Whipple (right posterior superior mesenteric artery-first approach), including a pylorus resection. All the patients underwent a pancreaticojejunal temporarily stented "duct-to-mucosa" end-to-side anastomosis, a hepaticojejunal end-to-side anastomosis and a side-to-side gastrojejunostomy. A standard lymphadenectomy was undertaken in all cases. The procedures were performed by the same surgical team. The mean operating time was about $5.5 \mathrm{~h}$, with a mean blood loss of $\sim 350 \mathrm{~mL}$, consistent with the reported values in other centers [14]. The patients were administered antibiotherapy up to the 3rd day after surgery, as far as no sign of infection was noted. All patients received a single dose of intraoperative Octreotide injection and intravenous $\mathrm{H} 2$ receptor antagonists each $12 \mathrm{~h}$ throughout the period of absent oral intake. Peritoneal drainage was measured daily. An early postoperative CT scan was performed at days $8-12$, followed by the removal of the draining tubes if no fistula or other complications were noticed.

\subsection{Clinically Relevant Pancreatic Fistula}

The pancreatic fistula is defined as a peritoneal drainage at more than 3 days after surgery of any quantity of fluid, with an amylase content of more than 3 times the seric amylase. The 2016 ISGPF classification and grading of pancreatic fistula makes a clear 
distinction between a biochemical leak (grade A) and clinically relevant fistula (grades B and C) [15]. Grade B pancreatic fistula requires adjusting the clinical management with antibiotherapy, nutritional supplements, Somatostatin analog and percutaneous drainage. Grade $C$ pancreatic fistula can result in sepsis, abdominal hemorrhage, multiorgan failure and even death.

Early postoperative CT images were assessed for the presence of fistulas, seen as fluid collections with or without gas, adjacent to the pancreatojejunal anastomosis [16].

\subsection{MDCT Acquisition Protocol}

For 55 of the 78 patients, the preoperative examination was performed using the 16-slice computed tomography (CT) scanner Somatom Sensation 16 (Siemens Medical Solutions, Erlangen, Germany) at a tube voltage of $140 \mathrm{kV}$, tube current of $150 \mathrm{~mA}$, standard convolution kernel, mean reconstruction field of view of $37 \mathrm{~cm}$ and matrix size of $512 \times 512$. The scanned volume extended from $2 \mathrm{~cm}$ above the diaphragm to the level of the pubic symphysis. Since our institution is a tertiary care center, 23 patients had preoperative CT acquisitions performed in other hospitals using nonstandard dynamic scan protocols. To minimize the effect of different slice thickness and reconstruction filters, we reformatted the images with a slice thickness of $5 \mathrm{~mm}$ and an increment of $4 \mathrm{~mm}$, using the diagnostic capabilities of the diagnostic console Intellispace Philips 10 available at our institution.

The preoperative scan was performed after the patients ingested $500 \mathrm{~mL}$ of diluted positive-contrast solution to facilitate the assessment of the anastomoses and the visualization of potential collections. When a nasogastric tube was present, it was clamped $1 \mathrm{~h}$ before the procedure.

Standard scan protocol included an unenhanced scan and an injection of iodine contrast media at a rate of $3 \mathrm{~mL} / \mathrm{s}$ with bolus tracker monitorization in the upper abdominal aorta, followed by two scans timed at $15 \mathrm{~s}$ and $40 \mathrm{~s}$ after the threshold of $150 \mathrm{HU}$. An additional delayed scan was performed at $3 \mathrm{~min}$ after the injection of the contrast in most patients.

\subsection{Investigated Parameters and Rationale:}

Our study investigated the possibility of using imaging-only parameters for the assessment of the risk of clinically relevant pancreatic fistula, objectively quantifiable on widely available preoperative $\mathrm{CT}$ scans without taking into account the intraoperative parameters (blood loss, operative time and vascular resections) or disease-specific parameters (TNM staging and neoadjuvant therapy).

Liver steatosis, as a possible indication of associated metabolic disorders, was assessed using the density of the liver during unenhanced preoperative scans. For each patient, we acquired three measurements with a region of interest (ROI) of $1 \mathrm{~cm}^{2}$ and noted the mean value.

The density of the pancreas is useful both for the evaluation of the degree of lipomatosis and for the identification of the degree of fibrosis. The pancreatic density was measured with a ROI of $1 \mathrm{~cm}^{2}$ placed at the estimated level of resection, excluding vascular structures, tumoral tissue, calcifications and ductal ectasias while minimizing partial volume effects from the surrounding structures; three measurements were performed, and we noted the mean value on the unenhanced scans and in the arterial, venous and late scans (Figure 1A). We also investigated the difference between the density of the pancreas in the arterial phase and delayed phase as a possible indicator of fibrosis of the pancreas but were limited by the availability of the scans (not part of the standard protocol) or the inconsistency of the timing of the delayed scans ( 3,4 and $10 \mathrm{~min})$.

The caliber of the main pancreatic duct was considered, as it is potentially useful in estimating the quality of the pancreaticojejunal -anastomosis. We noted the caliber of the duct at the estimated site of pancreatic resection (Figure 1B).

An estimated remnant pancreatic volume was considered, as it can potentially influence the risk of pancreatic fistula, patients at a high risk having a greater pancreatic volume, 
with a greater surface area of the pancreato-entero anastomosis. Volume estimation was performed using the volumetric tools in Intellispace 10 software (Philips) using a clipping plane at the left of the superior mesenteric vein (Figure 1C,D).
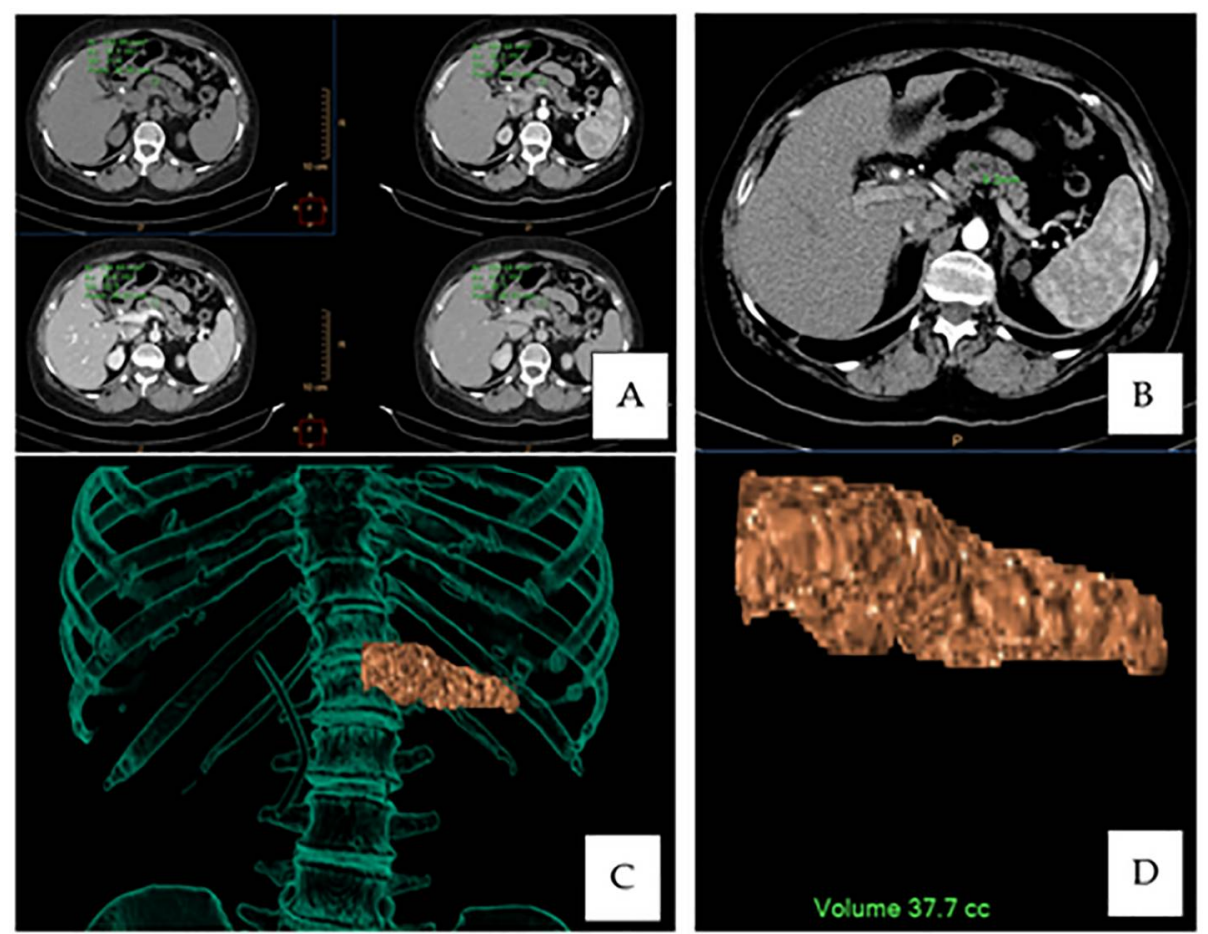

Figure 1. Parameter acquisitions: (A) Pancreatic density on a multisequence CT scan at the site of resection. (B) Main pancreatic duct measurement. (C,D) Volumetric rendering overlay and estimated pancreatic remnant volume calculation.

\subsection{Statistical Analysis}

The statistical analysis began by inspecting the continuous variables for the normality of distribution using the Shapiro-Wilk test $(p>0.05)$, histogram analysis and z score.

For the analysis of the correlation of the continuous numeric independent parameters with the clinically relevant pancreatic fistula (CRPF), we used a univariate analysis. Statistically significant independent parameters were used in the multiple logistic regression to evaluate the contribution of independent risk factors in the univariate analysis.

The predictive power of the models was analyzed using the area under the receiver operating characteristic curve (AUC) method. A concordance index $>0.8$ was considered reliable.

In all statistical tests, a value of $p<0.05$ was considered statistically significant.

All statistical tests were performed with SPSS version 23 (IBM Corp., Armonk, NY, USA).

To compare the strength of the proposed models, we first verified the correlation with the alternative fistula risk score (aFRS) [17] using Spearman's correlation and then assessed the difference between them using the DeLong test in MedCalc Statistical software version 20.006 (MedCalc Software Ltd., Ostend, Belgium).

\section{Results}

In total, 78 patients were enrolled in the study: 40 females (51.3\%) and 38 males $(48.7 \%)$, with a mean age of 59.33 years (SD 11.47) and a median body mass index (BMI) of 21 (IQR: 19-23) Kg/m² (Table 1). 
Table 1. Baseline characteristics of the retrospective group.

\begin{tabular}{|c|c|c|c|}
\hline Age, Mean (SD) & $59.33(11.47)$ & Disease, $n(\%)$ & \\
\hline Sex (male), $n(\%)$ & $38(48.7 \%)$ & Pancreatic ductal adenocarcinoma & $19(24.35)$ \\
\hline BMI, median (IQR) $\mathrm{Kg} / \mathrm{m}^{2}$ & $21(19-23)$ & Chronic pancreatitis & $7(8.97)$ \\
\hline ASA classification & & Neuroendocrine tumor & $4(5.12)$ \\
\hline $\mathrm{I}$ & $18(23.07 \%)$ & Cholangiocarcinoma & $6(7.69)$ \\
\hline II & $46(58.97 \%)$ & Ampullary carcinoma & $24(30.76)$ \\
\hline III-IV & $13(16.66 \%)$ & Duodenal carcinoma & $12(15.38)$ \\
\hline Comorbidity, $n(\%)$ & & Cystic neoplasms & $2(2.56)$ \\
\hline Cardiac & $16(21.79 \%)$ & Other & $5(6.41)$ \\
\hline Hypertension & $17(21.79 \%)$ & Pathology, $n(\%)$ & \\
\hline Pulmonary & $9(11.53 \%)$ & Malignant & $65(83.33)$ \\
\hline Diabetes mellitus & $12(15.38 \%)$ & Benign & $13(16.66)$ \\
\hline
\end{tabular}

BMI: Body Mass Index; IQR: Interquartile Range; ASA: American Society of Anaesthesiologists Physical Status.

Clinically relevant postoperative pancreatic fistula was observed in 22 patients $(28.2 \%)$. A correlation of the morphological parameters assessed on preoperative CT with the risk of clinically relevant pancreatic fistula is illustrated in Table 2.

Table 2. Univariate analysis of the independent factors and their association with clinically relevant pancreatic fistula (CRPF).

\begin{tabular}{|c|c|c|c|c|c|}
\hline \multirow[t]{2}{*}{ Independent Variable } & \multicolumn{2}{|c|}{$\begin{array}{c}\text { Non-Clinically Relevant } \\
\text { Pancreatic Fistula } \\
N=56\end{array}$} & \multicolumn{2}{|c|}{$\begin{array}{l}\text { Clinically Relevant } \\
\text { Pancreatic Fistula } \\
\qquad N=22\end{array}$} & \multirow[t]{2}{*}{$p$-Value } \\
\hline & Mean & SD & Mean & SD & \\
\hline Unenhanced density of the liver (HU) & 50.54 & SD 6.31 & 44.09 & 6.80 & 0.008 \\
\hline Pancreatic density (unenhanced) & 32.42 & 11.93 & 22.24 & 11.49 & 0.02 \\
\hline Pancreatic density (arterial) (HU) & 81.35 & 22.66 & 73.39 & 18.63 & 0.308 \\
\hline Pancreatic density (venous) (HU) & 79.23 & 18.29 & 67.55 & 19.66 & 0.087 \\
\hline Pancreatic density (delayed scan) (HU) & 61.28 & 16.55 & 48.67 & 18.05 & 0.045 \\
\hline $\begin{array}{l}\text { Difference between the arterial and delayed } \\
\text { density of the pancreas (HU) }\end{array}$ & 19.45 & 20.32 & 24.7 & 15.39 & 0.42 \\
\hline Main pancreatic duct diameter (mm) & 3.146 & 2.95 & 0.93 & 0.35 & 0.02 \\
\hline Total pancreatic volume $\left(\mathrm{cm}^{3}\right)$ & 76.69 & 31.49 & 91.31 & 26.68 & 0.185 \\
\hline Estimated remnant pancreatic volume $\left(\mathrm{cm}^{3}\right)$ & 34.87 & 12.35 & 46.37 & 10.39 & 0.01 \\
\hline
\end{tabular}

A total of five independent parameters were found to be significantly different in the group with CRPF compared to the non-CRPF group: the unenhanced density of the liver (UDL), the unenhanced pancreatic density (UPD), the delayed scan density of the pancreas, the main pancreatic duct (MPD) diameter and the estimated pancreatic remnant volume (EPRV). Due to the unavailability of the delayed scan of the pancreas in all patients and the differences in the scan protocols between different centers (the timing of the delayed scans varied at 3, 4 or 10 min after the injection of contrast, which could potentially alter the validity of the acquired values), we excluded this parameter from further analysis. Additionally, because of the low number of cases, we tried to restrict the model to three parameters.

Next, we verified the independence of the factors by analyzing the Pearson/Spearman's correlation of the factors, and we observed a correlation between the unenhanced density 
of the liver and the unenhanced density of the pancreas $(\mathrm{r}=0.29, p=0.07)$ and the main pancreatic duct diameter $\left(r_{s}=0.14, p=0.37\right)$, suggesting the need to exclude one of these parameters.

In the multiple logistic regression, we compared the predictive power of two models by using three factors, using both the MPD and the EPRV and differing in the third independent parameter. The predictive power of the model using the unenhanced pancreatic density preliminary proved to be higher than the one using the unenhanced density of the liver (92.3\% compared to $84.6 \%$ ). Given that marked pancreatic atrophy is frequent in pancreatic head cancers, we proceeded to investigate both models, with the second model potentially being used as an alternative in these patients.

Model 1 (using ERPV, MPD and UPD).

The logistic regression model predicted CRPF after pancreatectomy (Table 3, Figure 2). The risk of CRPF was estimated using the formula $y=1 /\left(1+\mathrm{e}^{-\mathrm{z}}\right)$, where $\mathrm{z}=-1.114+$ $0.155 \times(\mathrm{EPRV})-0.787 \times(\mathrm{MPD})-0.16 \times(\mathrm{UPD})$. A result of $>0.5$ estimated a significant risk for CRPF.

Table 3. Multivariate logistic regression for Model 1.

\begin{tabular}{cccccc}
\hline & Weight $(\beta)$ & Odds Ratio & $p$ & \multicolumn{2}{c}{ CI } \\
\hline & & & & Lower & Upper \\
\hline ERPV & 0.155 & 1.168 & 0.02 & 1.025 & 1.330 \\
\hline MPD & -0.787 & 0.455 & 0.02 & 0.235 & 0.881 \\
\hline UPD & -0.160 & 0.852 & 0.032 & 0.736 & 0.986 \\
\hline
\end{tabular}

Constant $\quad-1.114$

ERPV: the preoperatively estimated pancreatic remnant volume; MPD: the main pancreatic duct diameter; UPD: the values of unenhanced pancreatic density.

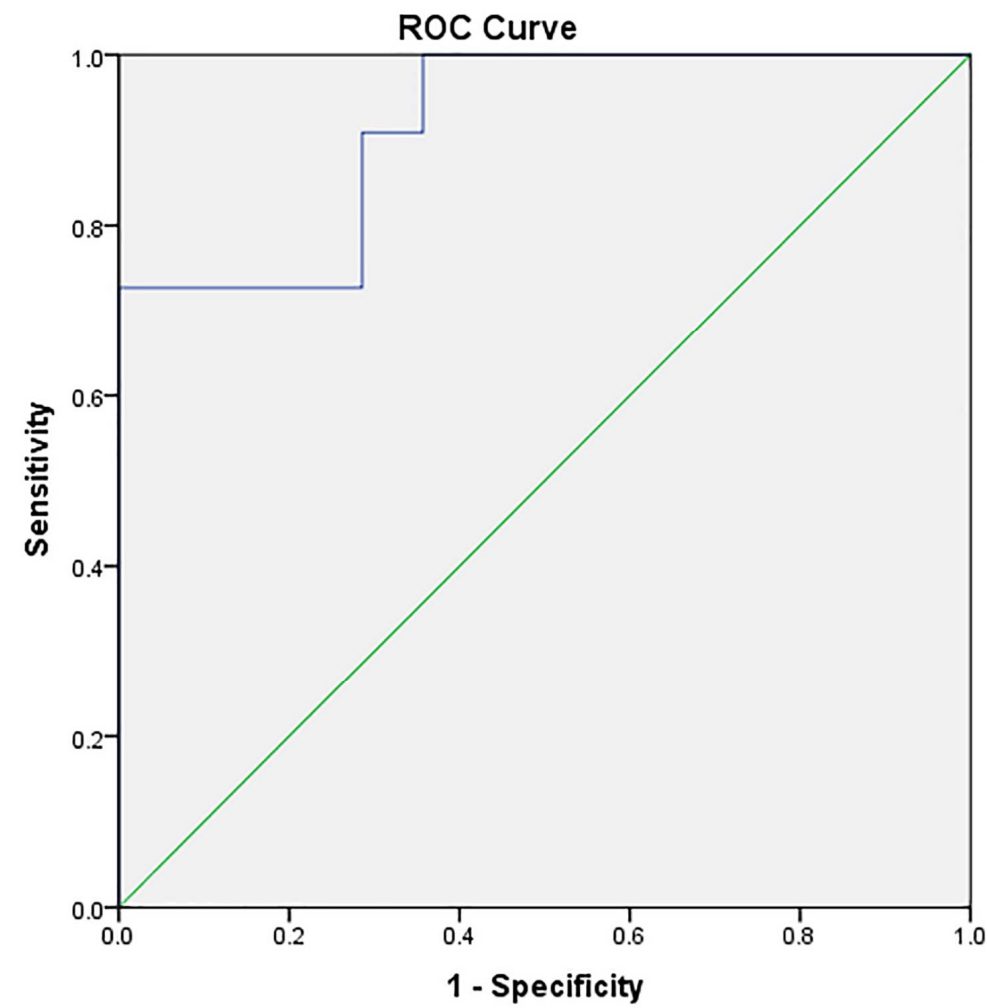

Figure 2. AUC for Model 1 using 3 parameters (preoperatively estimated pancreatic remnant volume, the main pancreatic duct diameter and the values of the unenhanced pancreatic density). 
To create a practical score, we proceeded to the estimation of the cutoff values for the different parameters. Using the Youden method, we established a cutoff value of $41 \mathrm{~cm}^{3}$ for the ERPV (with a sensibility of $81.8 \%$ and a specificity of $71.4 \%$ ) and $30 \mathrm{HU}$ for the unenhanced density of the pancreas (sensibility $90.0 \%$ and specificity of $71.4 \%$ ).

We checked the correlation of the BMI with the unenhanced pancreatic density, as an expression of the metabolic status, using Spearman's correlation and found a low correlation, although without statistical significance $\left(r_{s}=-0.155, p=0.167\right)$. Due to the non-gaussian distribution of the data for the pancreatic duct diameter, we proceeded to estimate the cutoff value for the main pancreatic duct by comparing the AUC of the model using different thresholds, starting from the maximum normal diameter $(3 \mathrm{~mm})$ and decreasing by $0.5 \mathrm{~mm}$ at each step, obtaining an optimum cutoff value of $2.5 \mathrm{~mm}$.

Based on the odds ratio, we allocated points to the score, as shown in Table 4.

Table 4. Proposed score for the assessment of the risk of pancreatic fistula (Model 1).

\begin{tabular}{cccccc}
\hline Variable & Cutoff Value & Weight $(\boldsymbol{\beta})$ & OR & \multicolumn{2}{c}{ Points Allocated } \\
\hline ERPV $\left(\mathrm{cm}^{3}\right)$ & $41 \mathrm{~cm}^{3}$ & 0.155 & 1.168 & $\begin{array}{c}\text { If }<41 \mathrm{~cm}^{3} \\
\text { If } \geq 41 \mathrm{~cm}^{3}\end{array}$ & $\begin{array}{c}0 \text { points } \\
2.5 \text { points }\end{array}$ \\
\hline $\begin{array}{c}\text { Unenhanced density } \\
\text { of the pancreas }(\mathrm{HU})\end{array}$ & $30 \mathrm{HU}$ & -0.160 & 0.852 & $\begin{array}{c}\text { If }>30 \mathrm{HU} \\
\text { If } \leq 30 \mathrm{HU}\end{array}$ & $\begin{array}{c}2 \text { points } \\
\text { points }\end{array}$ \\
\hline MPD diameter $(\mathrm{mm})$ & 2.5 & -0.787 & 0.455 & $\begin{array}{l}\text { If }>2.5 \mathrm{~mm} \\
\text { If } \leq 2.5 \mathrm{~mm}\end{array}$ & $\begin{array}{c}0 \text { points } \\
1 \text { points }\end{array}$ \\
\hline
\end{tabular}

ERPV: the preoperatively estimated pancreatic remnant volume; MPD: the main pancreatic duct diameter.

We scored 2.5 points for ERPV higher than $41 \mathrm{~cm}^{3}, 2$ points for UPD lower than $30 \mathrm{HU}$, 1 point for MPD diameter lower than $2.5 \mathrm{~mm}$ and 0 points for each of those conditions when they were not met.

We analyzed the distribution of the scores related to the clinically relevant pancreatic fistula (Figure 3) and found that a score greater than 3 could identify patients at risk for CRPF with an AUC of 0.92 (95\% CI: 0.828-1, sensibility 90.9\% and specificity 67.9\%) and with a positive predicting value of $47.82 \%$ and a negative predicting value of $100 \%$, which suggests the score could correctly identify low-risk patients, although it could not accurately identify high-risk patients.

Using a cutoff score of 4.5 provided a slightly lower AUC of 0.846 (95\% CI: 0.694-0.941), sensibility $72.72 \%$ and specificity $96.4 \%$ ) but with a higher positive predictive value $(88.88 \%)$ and an acceptable negative predictive value (90\%), suggesting a good accuracy for the detection of high-risk patients. The mean probability of CRPF for patients with a score of more than or equal to 4.5 was $76.4 \%$ (95\% CI: 7.35-21.3), compared with $13.84 \%$ (95\% CI: 58.01-94.92) for scores below 4.5.

A Spearman's correlation comparison of the score derived from Model 1 to the aFRS showed a good correlation $\left(r_{s}=0.633, p<0.001\right)$. Next, we performed the DeLong test, showing a minor improvement of the AUC using our score compared to the aFRS (AUC 0.846 vs. 0.808$)$ but without being statistically significantly better $(p=0.661)$ (Figure 4$)$. 


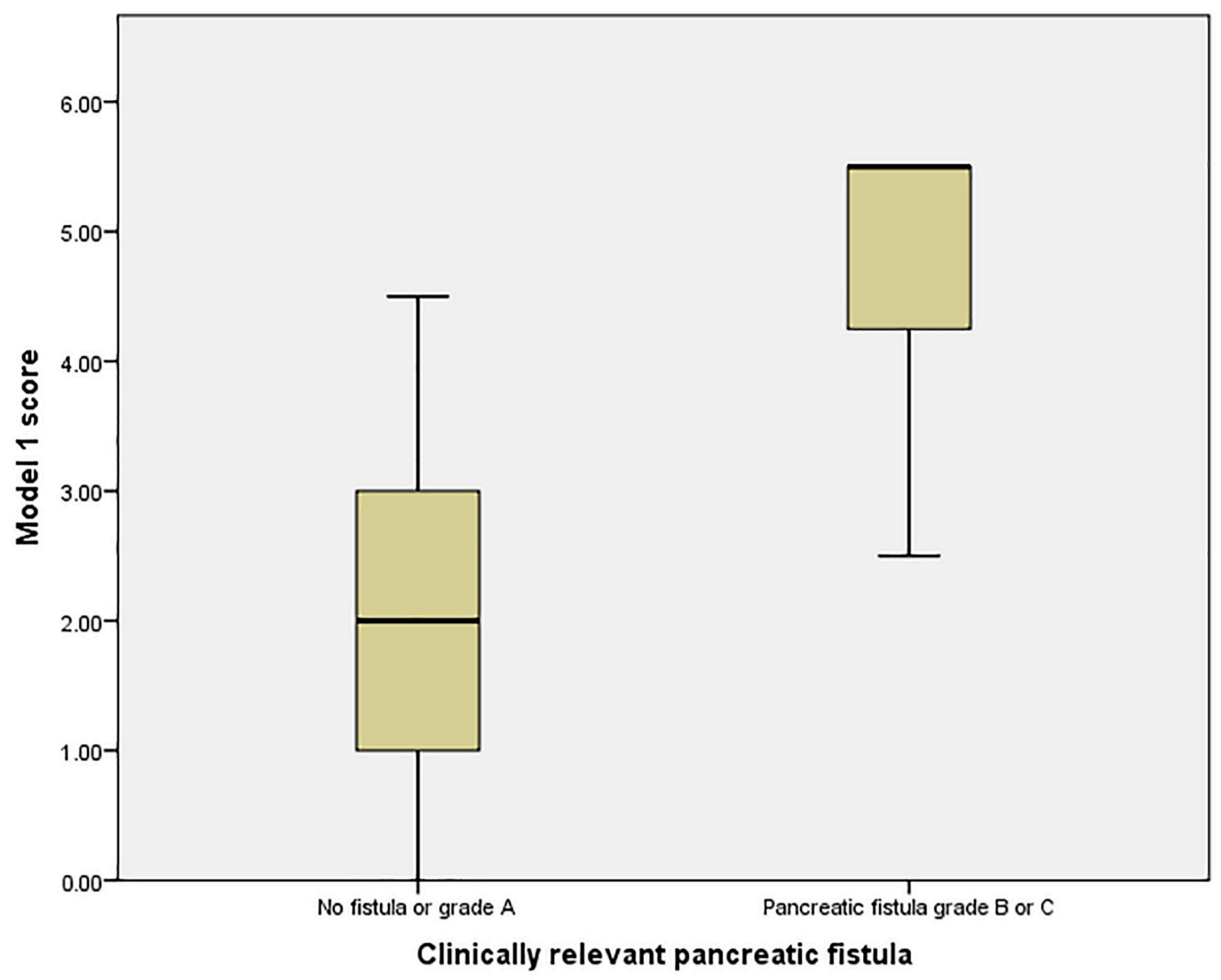

Figure 3. Risk groups for the clinically relevant pancreatic fistula (CRPF) in Model 1.

\begin{tabular}{|c|c|c|c|c|}
\hline \multicolumn{5}{|c|}{ Comparison of ROC curves } \\
\hline Variable 1 & \multicolumn{4}{|c|}{ aFRS (alternative Fistula Risk Score) } \\
\hline Variable 2 & \multicolumn{4}{|c|}{ Model 1 score with a threshold of 4.5} \\
\hline Classification variable & \multicolumn{4}{|c|}{ CRPF (clinically relevant pancreatic fistula) } \\
\hline Sample size & \multicolumn{4}{|r|}{78} \\
\hline Positive group a & \multicolumn{4}{|r|}{$22(28.21 \%)$} \\
\hline Negative group ${ }^{b}$ & \multicolumn{4}{|r|}{$56(71.79 \%)$} \\
\hline \multicolumn{5}{|l|}{$\begin{array}{l}\mathrm{a} \text { CRPF }=1 \\
{ }^{b} \mathrm{CRPF}=0\end{array}$} \\
\hline \multicolumn{2}{|l|}{ Variable } & AUC & SEa & $95 \% \mathrm{Cl}{ }^{\mathrm{b}}$ \\
\hline \multicolumn{2}{|l|}{ aFRS } & 0.808 & 0.104 & 0.651 to 0.916 \\
\hline \multicolumn{2}{|c|}{ Model 1 score with a threshold of 4.5} & 0.846 & 0.0726 & 0.694 to 0.941 \\
\hline \multicolumn{5}{|l|}{$\begin{array}{l}\text { DeLong et al. } 1988 \\
\text { binomial exact }\end{array}$} \\
\hline \multicolumn{5}{|c|}{ Pairwise comparison of ROC curves } \\
\hline \multicolumn{5}{|c|}{ aFRS - Model 1 score with a threshold of 4.5} \\
\hline \multicolumn{3}{|c|}{ Difference between areas } & \multicolumn{2}{|r|}{0.0373} \\
\hline \multicolumn{3}{|l|}{ Standard Error a } & \multicolumn{2}{|r|}{0.0853} \\
\hline \multicolumn{3}{|c|}{$95 \%$ Confidence Interval } & \multicolumn{2}{|r|}{-0.130 to 0.205} \\
\hline \multicolumn{3}{|l|}{ z statistic } & \multicolumn{2}{|r|}{0.438} \\
\hline \multicolumn{3}{|l|}{ Significance level } & \multicolumn{2}{|r|}{$p=0.6615$} \\
\hline
\end{tabular}

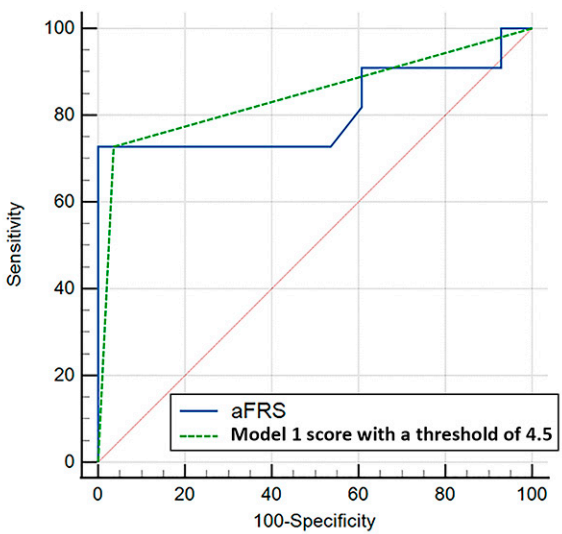

Figure 4. AUC comparison of aFRS and the score in Model 1 for the prediction of CRPF.

Model 2 (using ERPV, MPD and UDL).

The risk of CRPF in this model was calculated using the formula $y=1 /\left(1+\mathrm{e}^{-\mathrm{z}}\right)$, where $\mathrm{z}=7.219+0.138 \times(\mathrm{EPRV})-0.870 \times(\mathrm{MPD})-0.256 \times(\mathrm{UDL})$ (Table 5, Figure 5). A result of $>0.5$ estimated a significant risk for CRPF. 
Table 5. Multivariate logistic regression for Model 2.

\begin{tabular}{cccccc}
\hline & Weight $(\beta)$ & Odds Ratio & $p$ & \multicolumn{2}{c}{ CI } \\
\hline ERPV & & & & Lower & Upper \\
\hline MPD & 0.138 & 1.148 & 0.026 & 1.017 & 1.295 \\
\hline ULD & -0.870 & 0.419 & 0.032 & 0.189 & 0.929 \\
\hline Constant & -0.256 & 0.774 & 0.032 & 0.635 & 0.944 \\
\hline
\end{tabular}

ERPV: the preoperatively estimated pancreatic remnant volume; MPD: the main pancreatic duct diameter; ULD: the unenhanced liver density.

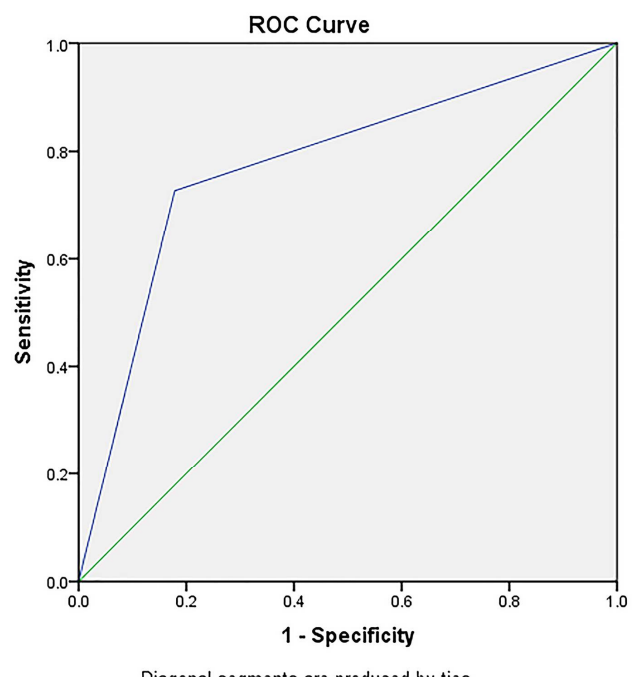

Figure 5. AUC for Model 2 using 3 parameters (the preoperatively estimated pancreatic remnant volume, the main pancreatic duct diameter, the unenhanced liver density).

We calculated the cutoff value for the UDL using the Youden method at $45 \mathrm{HU}$ (sensibility 76.4\% and specificity 78.6\%). Hepatic steatosis correlated with the BMI with a correlation coefficient $r_{\mathrm{S}}=-0.130$ and $p=0.334$.

Based on the odds ratio, we allocated points to the alternative score, as shown in Table 6.

Table 6. Alternative score for the assessment of the risk of CRPF using Model 2.

\begin{tabular}{cccccc}
\hline Variable & Cutoff Value & Weight $(\beta)$ & OR & \multicolumn{2}{c}{ Points Allocated } \\
\hline ERPV $\left(\mathrm{cm}^{3}\right)$ & $41 \mathrm{~cm}^{3}$ & 0.138 & 1.148 & $\begin{array}{l}\text { If }<41 \mathrm{~cm}^{3} \\
\text { If } \geq 41 \mathrm{~cm}^{3}\end{array}$ & $\begin{array}{c}0 \text { points } \\
3 \text { points }\end{array}$ \\
\hline $\begin{array}{c}\text { Unenhanced density } \\
\text { of the liver }(\mathrm{HU})\end{array}$ & $45 \mathrm{HU}$ & -0.256 & 0.774 & $\begin{array}{l}\text { If }>45 \mathrm{HU} \\
\text { If } \leq 45 \mathrm{HU}\end{array}$ & $\begin{array}{l}0 \text { points } \\
2 \text { points }\end{array}$ \\
\hline MPD diameter $(\mathrm{mm})$ & 2.5 & -0.870 & 0.419 & $\begin{array}{l}\text { If }>2.5 \mathrm{~mm} \\
\text { If } \leq 2.5 \mathrm{~mm}\end{array}$ & $\begin{array}{l}0 \text { points } \\
1 \text { points }\end{array}$ \\
\hline
\end{tabular}

ERPV: the preoperatively estimated pancreatic remnant volume; MPD: the main pancreatic duct.

In Model 2, we scored 3 points for ERPV higher than $41 \mathrm{~cm}^{3}, 2$ points for UDL lower than $45 \mathrm{HU}, 1$ point for a MPD diameter lower than $2.5 \mathrm{~mm}$ and 0 points for each of those conditions when they were not met.

A risk stratification analysis (Figure 6) showed that a score greater than 4 produced an AUC of 0.774 (95\% CI $0.599-0.850)$ with a sensibility of $72.7 \%$ and a specificity of $82.1 \%$, a positive predictive value of $61.5 \%$ and a negative predictive value of $88.46 \%$. The mean probability for CRPF for patients with a score $\geq 4$ was $60 \%$ (95\% CI 37.87-82.13), compared to $12.3 \%(95 \%$ CI $3.79-20.81)$ for scores $<4$. 
The correlation of the score model with the aFRS was found to be significant $\left(\mathrm{r}_{\mathrm{s}}=0.524\right.$, $p=0.001)$. Comparison with the aFRS using the DeLong test showed no improvement over the aFRS $(p=0.715)$ (Figure 7).

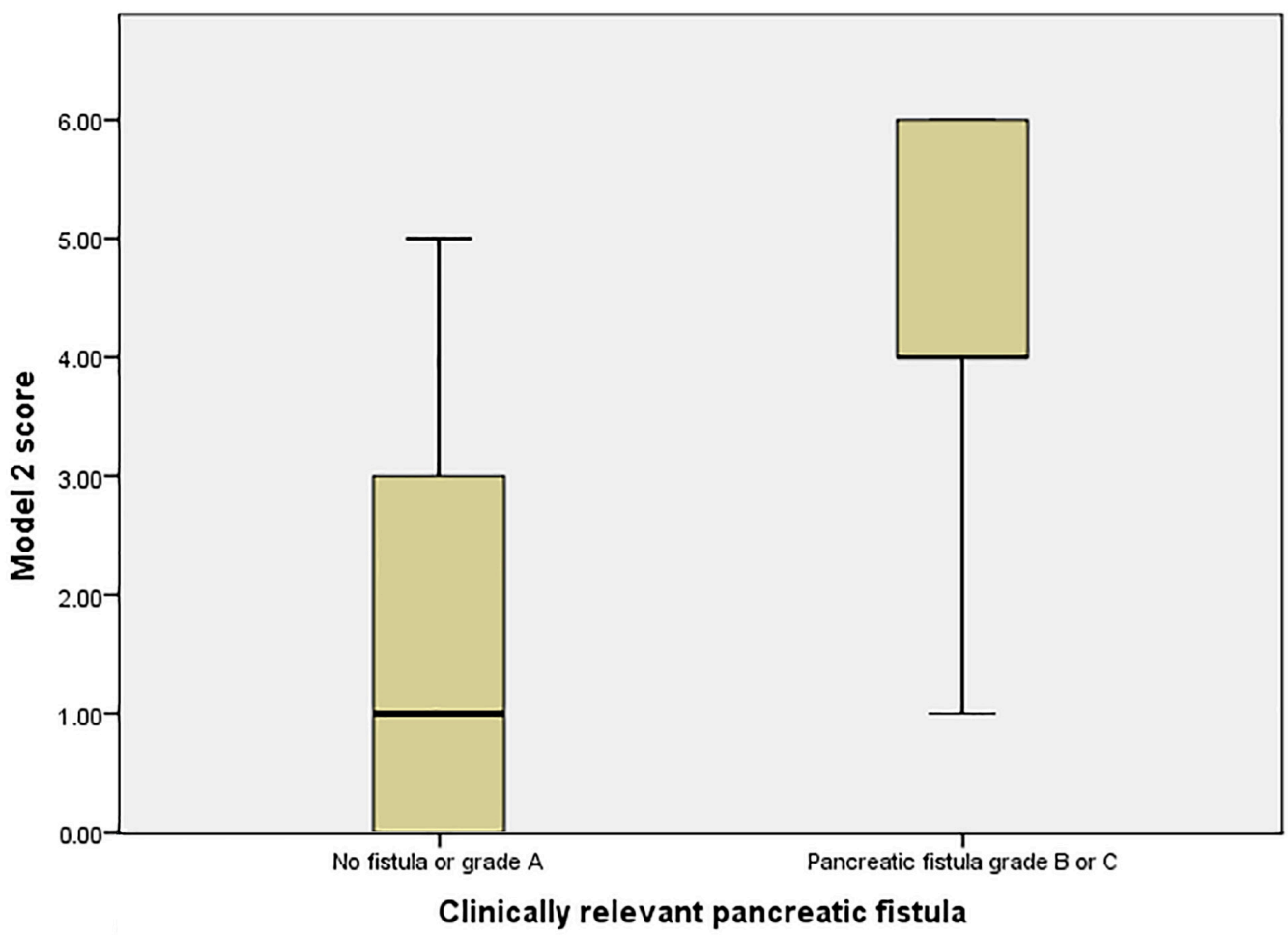

Figure 6. Risk groups for CRPF in Model 2.

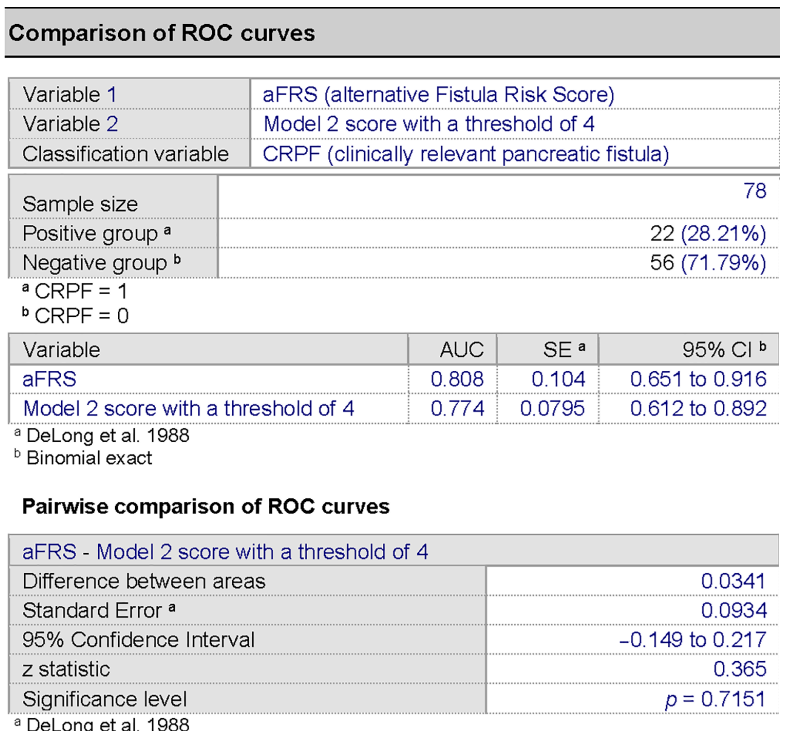

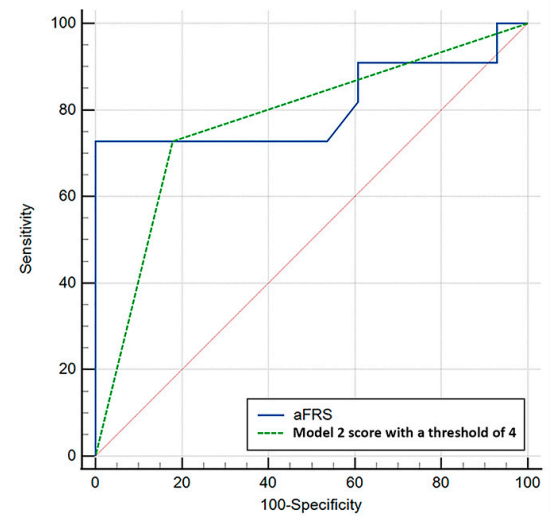

Figure 7. Comparison of the alternative fistula score and our proposed model for the prediction of CRPF.

\section{Discussion}

Predicting the risk of pancreatic fistula remains controversial, with multiple proposed scores in the literature when using pre-, intra- and postoperative parameters with different accuracy levels (AUC between 0.656 and 0.806) [17-23]. 
Although some studies noted the influence of the surgical technique on the risk of pancreatic fistula [24], other authors stated that the type of anastomosis and the placement of the pancreatic stent did not influence the risk of pancreatic fistula [25].

Our study focused solely on evaluating the contribution of independent imaging parameters derived from preoperative contrast-enhanced computer tomography scans and confirmed a number of parameters correlating with the risk of CRPF: the unenhanced density of the liver (the degree of liver steatosis), the unenhanced density of the pancreas (pancreatic steatosis), the delayed density of the pancreas (a parameter that we further excluded because of the inconsistence and nonuniformity in the timing of the sequence between examinations), the main pancreatic duct diameter and the estimated remnant pancreatic volume. An investigation of the correlation of the metabolic status of the patient (expressed through the BMI) with liver/pancreatic steatosis showed only a mild correlation but did not provide statistically significant results, which could be a consequence of both the small sample size and the particularities of the cohort, as multiple patients had a diagnosis of chronic pancreatitis, caused by alcohol consumption, that can alter the measured density of the pancreas [26].

We proposed two scores that can be derived from a preoperatively obtained unenhanced CT scan of the abdomen using the following parameters: the unenhanced density of the pancreas at the estimated site of resection (to the left of the superior mesenteric vein) using a cutoff value of $30 \mathrm{HU}$, the main pancreatic duct diameter at the estimated site of anastomosis using a cutoff value of $2.5 \mathrm{~mm}$, the estimated remnant pancreatic volume using a cutoff value of $41 \mathrm{~cm}^{3}$ and the unenhanced density of the liver using a cutoff value of $45 \mathrm{HU}$. Although the score using Model 1 (UPD, MPD and ERPV) showed a good performance compared to the aFRS, we derived a second score based on Model 2 (UDL, MPD and ERPV) that did not produce the same performance level but can be a useful alternative in patients with marked atrophy of the pancreas (for whom the confident and reproductible measurement of the unenhanced pancreatic density can be difficult), a frequent situation in pancreatic head neoplasms.

Both scores showed a good sensibility and specificity for the prediction of clinically relevant pancreatic fistula. Although our data showed a mild improvement in the AUC analysis compared to the aFRS in the first model, it did not have a significant improvement when analyzed with the DeLong test, a situation that could be related to the intrinsic limitations of the test when the model was developed and deployed on the same dataset [27].

The good negative predicting value of the scores could be used in the selection of low-risk patients, for whom the complication of mitigation strategies could be adjustedopting, for example, for a no-drain strategy-especially considering the complications that may occur because of the draining tubes [28,29].

The proposed scores use objective, CT-based independent parameters, irrespective of the intraoperative parameters and histology, and might be an early and useful tool to tailor surgical procedures and postoperative strategies.

The technique employed for the establishment of the cutoff values had a limitation, as they are directly derived from the test set. In our dataset, the threshold value for the pancreatic duct size was found similar to the one found by Xia et al. [19]. Additionally, our study obtained a different cutoff value for the estimated remnant pancreatic volume as compared to Miyamoto et al. [30].

Another shortcoming of our study was the small number of patients, necessitating a further study to validate the proposed CT-based pancreatic fistula risk score.

\section{Conclusions}

Clinically relevant pancreatic fistula can be predicted using preoperative CT scan of the abdomen when using the following parameters: estimated remnant pancreatic volume, main pancreatic duct diameter, pancreatic steatosis and liver steatosis. We formulated two score-based models, the second one having a lower accuracy but useful as an alternative for patients with marked atrophy of the pancreas. 
Author Contributions: Conceptualization, M.L.S. and C.L.; data curation, M.L.S. and C.L.; formal analysis, M.L.S.; investigation, F.M., L.G., D.N., A.M.T., M.Z., V.N., R.C., M.B., O.L. and F.C.; methodology, C.L.; project administration, M.L.S.; supervision, C.L.U.; writing—original draft, M.L.S., C.L.U. and C.L. and writing-review and editing, C.L.U. and C.L. All authors have read and agreed to the published version of the manuscript.

Funding: This research received no external funding.

Institutional Review Board Statement: The study was conducted according to the guidelines of the Declaration of Helsinki and approved by the Institutional Review Board of Gr. T. Popa University of Medicine and Pharmacy Iasi (No. 19802, approved date 14 April 2020).

Informed Consent Statement: Informed consent was obtained from all subjects involved in the study.

Data Availability Statement: The data presented in this study are available on request from the corresponding authors.

Conflicts of Interest: The authors declare no conflict of interest.

\section{References}

1. Malleo, G.; Pulvirenti, A.; Marchegiani, G.; Butturini, G.; Salvia, R.; Bassi, C. Diagnosis and management of postoperative pancreatic fistula. Langenbeck's Arch. Surg. 2014, 399, 801-810. [CrossRef]

2. Tien, Y.W.; Lee, P.H.; Yang, C.Y.; Ho, M.C.; Chiu, Y.F. Risk factors of massive bleeding related to pancreatic leak after pancreaticoduodenectomy. J. Am. Coll. Surg. 2005, 201, 554-559. [CrossRef]

3. Gouma, D.J.; Van Geenen, R.C.I.; Van Gulik, T.M.; De Haan, R.J.; De Wit, L.T.; Busch, O.R.C.; Obertop, H. Rates of complications and death after pancreaticoduodenectomy: Risk factors and the impact of hospital volume. Ann. Surg. 2000, 232, 786-795. [CrossRef]

4. Hashimoto, Y.; Traverso, L.W. Incidence of pancreatic anastomotic failure and delayed gastric emptying after pancreatoduodenectomy in 507 consecutive patients: Use of a web-based calculator to improve homogeneity of definition. Surgery 2010, 147, 503-515. [CrossRef]

5. Lin, J.W.; Cameron, J.L.; Yeo, C.J.; Riall, T.S.; Lillemoe, K.D. Risk factors and outcomes in postpancreaticoduodenectomy pancreaticocutaneous fistula. J. Gastrointest. Surg. 2004, 8, 951-959. [CrossRef] [PubMed]

6. Mathur, A.; Pitt, H.A.; Marine, M.; Saxena, R.; Schmidt, C.M.; Howard, T.J.; Nakeeb, A.; Zyromski, N.J.; Lillemoe, K.D. Fatty pancreas: A factor in postoperative pancreatic fistula. Ann. Surg. 2007, 246, 1058-1064. [CrossRef] [PubMed]

7. Harada, N.; Ishizawa, T.; Inoue, Y.; Aoki, T.; Sakamoto, Y.; Hasegawa, K.; Sugawara, Y.; Tanaka, M.; Fukayama, M.; Kokudo, N. Acoustic radiation force impulse imaging of the pancreas for estimation of pathologic fibrosis and risk of postoperative pancreatic fistula. J. Am. Coll. Surg. 2014, 219, 887-894.e5. [CrossRef]

8. Tajima, Y.; Matsuzaki, S.; Furui, J.; Isomoto, I.; Hayashi, K.; Kanematsu, T. Use of the time-signal intensity curve from dynamic magnetic resonance imaging to evaluate remnant pancreatic fibrosis after pancreaticojejunostomy in patients undergoing pancreaticoduodenectomy. Br. J. Surg. 2004, 91, 595-600. [CrossRef] [PubMed]

9. Takahashi, N.; Fletcher, J.G.; Hough, D.M.; Fidler, J.L.; Kawashima, A.; Mandrekar, J.N.; Chari, S.T. Autoimmune pancreatitis: Differentiation from pancreatic carcinoma and normal pancreas on the basis of enhancement characteristics at dual-phase CT. Am. J. Roentgenol. 2009, 193, 479-484. [CrossRef]

10. Kim, S.Y.; Kim, H.; Cho, J.Y.; Lim, S.; Cha, K.; Lee, K.H.; Kim, Y.H.; Kim, J.H.; Yoon, Y.-S.; Han, H.-S.; et al. Quantitative assessment of pancreatic fat by using unenhanced CT: Pathologic correlation and clinical implications. Radiology 2014, 271, 104-112. [CrossRef]

11. Hashimoto, Y.; Sclabas, G.M.; Takahashi, N.; Kirihara, Y.; Smyrk, T.C.; Huebner, M.; Farnell, M.B. Dual-Phase Computed Tomography for Assessment of Pancreatic Fibrosis and Anastomotic Failure Risk Following Pancreatoduodenectomy. J. Gastrointest. Surg. 2011, 15, 2193-2204. [CrossRef]

12. Kajiwara, T.; Sakamoto, Y.; Morofuji, N.; Nara, S.; Esaki, M.; Shimada, K.; Kosuge, T. An analysis of risk factors for pancreatic fistula after pancreaticoduodenectomy: Clinical impact of bile juice infection on day 1. Langenbeck's Arch. Surg. 2010, 395, 707-712. [CrossRef]

13. Kanda, M.; Fujii, T.; Suenaga, M.; Takami, H.; Hattori, M.; Inokawa, Y.; Yamada, S.; Nakayama, G.; Sugimoto, H.; Koike, M.; et al. Estimated pancreatic parenchymal remnant volume accurately predicts clinically relevant pancreatic fistula after pancreatoduodenectomy. Surgery 2014, 156, 601-610. [CrossRef] [PubMed]

14. Cameron, J.L.; Riall, T.S.; Coleman, J.; Belcher, K.A. One thousand consecutive pancreaticoduodenectomies. Ann. Surg. 2006, 244, 10-15. [CrossRef]

15. Bassi, C.; Marchegiani, G.; Dervenis, C.; Sarr, M.; Abu Hilal, M.; Adham, M.; Allen, P.; Andersson, R.; Asbun, H.J.; Besselink, M.G.; et al. The 2016 update of the International Study Group (ISGPS) definition and grading of postoperative pancreatic fistula: 11 Years After. Surgery 2017, 161, 584-591. [CrossRef] [PubMed] 
16. Hashimoto, M.; Koga, M.; Ishiyama, K.; Watarai, J.; Shibata, S.; Sato, T.; Yamamoto, Y. CT features of pancreatic fistula after pancreaticoduodenectomy. Am. J. Roentgenol. 2007, 188, W323-W327. [CrossRef] [PubMed]

17. Mungroop, T.H.; Van Rijssen, L.B.; Van Klaveren, D.; Smits, F.J.; Van Woerden, V.; Linnemann, R.J.; de Pastena, M.; Klompmaker, S.; Marchegiani, G.; Ecker, B.L.; et al. Alternative Fistula Risk Score for Pancreatoduodenectomy (a-FRS): Design and International External Validation. Ann. Surg. 2019, 269, 937-943. [CrossRef]

18. Callery, M.P.; Pratt, W.B.; Kent, T.S.; Chaikof, E.L.; Vollmer, C.M. A prospectively validated clinical risk score accurately predicts pancreatic fistula after pancreatoduodenectomy. J. Am. Coll. Surg. 2013, 216, 1-14. [CrossRef] [PubMed]

19. Xia, W.; Zhou, Y.; Lin, Y.; Yu, M.; Yin, Z.; Lu, X.; Hou, B.; Jian, Z. A predictive risk scoring system for clinically relevant pancreatic fistula after pancreaticoduodenectomy. Med. Sci. Monit. 2018, 24, 5719-5728. [CrossRef]

20. Gaujoux, S.; Cortes, A.; Couvelard, A.; Noullet, S.; Clavel, L.; Rebours, V.; Lévy, P.; Sauvanet, A.; Ruszniewski, P.; Belghiti, J. Fatty pancreas and increased body mass index are risk factors of pancreatic fistula after pancreaticoduodenectomy. Surgery 2010, 148, 15-23. [CrossRef]

21. Wellner, U.F.; Kayser, G.; Lapshyn, H.; Sick, O.; Makowiec, F.; Höppner, J.; Hopt, U.T.; Keck, T. A simple scoring system based on clinical factors related to pancreatic texture predicts postoperative pancreatic fistula preoperatively. HPB 2010, 12, 696-702. [CrossRef]

22. Roberts, K.J.; Hodson, J.; Mehrzad, H.; Marudanayagam, R.; Sutcliffe, R.P.; Muiesan, P.; Isaac, J.; Bramhall, S.R.; Mirza, D.F. A preoperative predictive score of pancreatic fistula following pancreatoduodenectomy. HPB 2014, 16, 620-628. [CrossRef]

23. Yamamoto, Y.; Sakamoto, Y.; Nara, S.; Esaki, M.; Shimada, K.; Kosuge, T. A preoperative predictive scoring system for postoperative pancreatic fistula after pancreaticoduodenectomy. World J. Surg. 2011, 35, 2747-2755. [CrossRef]

24. Wada, K.; Traverso, L.W. Pancreatic anastomotic leak after the Whipple procedure is reduced using the surgical microscope. Surgery 2006, 139, 735-742. [CrossRef] [PubMed]

25. Harrell, F.E.; Califf, R.M.; Pryor, D.B.; Lee, K.L.; Rosati, R.A. Evaluating the Yield of Medical Tests. J. Am. Med. Assoc. 1982, 247, 2543-2546. [CrossRef]

26. Sharma, P.; Arora, A. Clinical presentation of alcoholic liver disease and non-alcoholic fatty liver disease: Spectrum and diagnosis. Transl. Gastroenterol. Hepatol. 2020, 5, 19. [CrossRef] [PubMed]

27. Demler, O.V.; Pencina, M.J.; D'Agostino, R.B. Misuse of DeLong test to compare AUCs for nested models. Stat. Med. 2012, 31, 2577-2587. [CrossRef]

28. Witzigmann, H.; Diener, M.K.; Kißenkötter, S.; Rossion, I.; Bruckner, T.; Werner, B.; Pridöhl, O.; Radulova-Mauersberger, O.; Lauer, H.; Knebel, P.; et al. No need for routine drainage after pancreatic head resection: The dual-center, randomized, controlled PANDRA trial (ISRCTN04937707). Ann. Surg. 2016, 264, 528-537. [CrossRef]

29. Bassi, C.; Molinari, E.; Malleo, G.; Crippa, S.; Butturini, G.; Salvia, R.; Talamini, G.; Pederzoli, P. Early versus late drain removal after standard pancreatic resections: Results of a prospective randomized trial. Ann. Surg. 2010, 252, 207-214. [CrossRef] [PubMed]

30. Miyamoto, R.; Oshiro, Y.; Sano, N.; Inagawa, S.; Ohkohchi, N. Three-Dimensional Remnant Pancreatic Volumetry Predicts Postoperative Pancreatic Fistula in Pancreatic Cancer Patients after Pancreaticoduodenectomy. Gastrointest. Tumors 2018, 5, 90-99. [CrossRef] 